\title{
Utilization of Dolutegravir and Lamivudine as Combination as A Single Tablet in Therapy of HIV-1 Infection
}

\author{
Kulvinder Kochar Kaur ${ }^{1 *}$ Gautam Allahbadia ${ }^{2}$ and Mandeep Singh ${ }^{3}$ \\ ${ }^{1}$ Scientific Director, Centre for Human Reproduction, Jalandhar, Punjab, India \\ ${ }^{2}$ Scientific Director, Ex-Rotunda-A Centre for Human reproduction, Bandra, Mumbai, \\ India \\ ${ }^{3}$ Neurology, Consultant Neurologist, Swami Satyanand Hospital, Jalandhar, Punjab, \\ India \\ *Corresponding Author: Kulvinder Kochar Kaur, Scientific Director, Centre for \\ Human Reproduction, Jalandhar, Punjab, India.
}

DOI: 10.31080/ASMI.2020.03.0526
Received: January 17, 2020

Published: February 14, 2020

(C) All rights are reserved by Kulvinder

Kochar Kaur., et al.

\begin{abstract}
Marked advances have been done regarding drug development for the therapy of HIV-1 - infection. With the formulation of Dolutegravir plus Lamivudine as combination Antiretroviral- therapy (cART) into single tablet regimens till the formation of long acting Antiretroviral (ARV) drug formulations, the therapy of HIV has become much more tolerable and will become even further tolerable as compared to initiation of therapy with zovidudine in 1987. Additionally, there is concentration on decreasing both short as well as long term toxicities and in same time sustaining heavy effectiveness. The main focus of this article is the $1^{\text {st }} 2$ drug regimen that has been approved for therapy by FDA for treatment naïve people residing with HIV-1(PLWH), that comprises of the integrase inhibitor Dolutegravir (DTG) as well as the nucleoside reverse transcriptase inhibitors (NRTIs) Lamivudine (3TC). The chemical composition, as well as pharmacodynamics, pharmacokinetics, as well as clinical trial data on safety) as well as effectiveness of DTG/3TC are discussed. Further emphasis on use of DTG/3TC in the frame of existing scenario and emerging ARV possibilities is further touched.
\end{abstract}

Keywords: HIV-1 - Infection; Cart; Dolutegravir (DTG; Lamivudine (3TC); ARV

\section{Introduction}

Lot of progress has been made in the formation of combined antiretroviral therapy (cART) for treating HIV infection following the approval of the $1^{\text {st }}$ antiretroviral drug, zidovudine in 1987. Following that drugs with >potency as well as efficacy and better tolerance of regimens that need less dose frequency and/or that get coformulated into a single tablet regimens (STR) has led to get a revolution in HIV therapy and allowed marked increase in life expectancy in people living with HIV (PLWH).

The treatment plans used at present are supported by Guidelines formed by US Department for Health and Human Services (DHHS) as well as International AIDS Society (IAS) are starting
HIV therapy with 2 nucleoside /nucleotide reverse transcriptase inhibitors (NRTIs) either combined with an integrase strand transfer inhibitor(INSTI), a protease inhibitor (PI) or a non nucleotide reverse transcriptase inhibitors (NNRTI) with INSTI the one having edge, while rest kept as alternative drugs for particular conditions when taken continuously and we have $>10$ STRs now [1-4].

One method of making therapy simple and keeping related toxicities to the least is utilization of dual ARV regimens (DTRs) which have been analyzed for early as well as maintainance treatment for PLWH [5,6]. Different efficacy of these studies have demonstrated different effectiveness but some of these regimen constructs correlated with a > risk of therapy emerging drug resistance along with 
reduced effectiveness in persons with high viral loads as well as / or low CD4 counts [7-9] The regimen that demonstrated adequate effectiveness and final approval by USFDA is dolutegravir/rilprivine but that is utilized for maintainance of suppression of virus in patients who had been therapy experienced [10].

Those who are treatment-naive persons have been evaluated where 2 drug to 3 drug regimens were compared. In an open label randomised study where 753 treatment-naive patients receiving ritonavir(r)-boosted lopinavir (LPV/r) or efavirenz (EFV), each combined with 2 NRTIs (standard of care[SOC]) to the NRTIssparing combination- EFV/ LPV/r, revealed that the effectiveness of dual regimens was same as the EFV/2 NRTIs arm but had > therapy emerging drug resistance $[6,11,12]$. Another experiment demonstrated that the 2 drugs, LPV combined with 3TC had similar effectiveness but better tolerance as compared to LPV/2 NRTIs in ARV naive persons [13].

In the same way dolutegravir (DTG) combined with 3 TC (DTG/3TC has been evaluated deeply as a 2 drug regimen having favourable properties like best tolerance with great resistance barrier (DTG). This is the $1^{\text {st }} 2$-drug STRs that got approval for PLWH, marketed under DOVATO name by Glaxo-Smith Kline, Research Triangle Park, NC USA) [14,15].

\section{Details of dolutegravir/lamivudine STR (DTG/3TC)}

It is a 2 drug combination of DTG, which is an INSTI and 3TC, being an NRTI, and has approval as a total regimen for therapy for HIV-1 infection in ARV naive adults not having genotypic resistance to separate ARV constituents [16].

In last 2 decades cART utilized 3drug regimen as original therapy for HIV therapy. DTG/3TC had to face competition in market of $>50$ approved medicines, many being coformulated drugs. But DTG/3TC is only the $2^{\text {nd }}$ approved 2 drug STRs and is the $1^{\text {st }}$ one approved for treatment-naive PLWH [17].

\section{Chemistry}

The single tablet has $300 \mathrm{mg}$ of Lamivudine and $50 \mathrm{mg}$ of dolutegravir (i.e equivalent to $52.6 \mathrm{mg}$ dolutegravir sodium) as active components. Inactive ingredients are K29/32, sodium starch glycolate, sodium starch glycolate, sodium stearyl fumarate magnesium stearate, mannitol and microcrystalline cellulose, hypromellose, polyethylene glycol, titanium dioxide in the tablets coat. This tablet is made for oral intake [15].

\section{Dolutegravir (DTG)}

DTG sodium represents a monocarboxylic acid amide as well as organic heterocyclic compound having a chemical formula sodium (4R,12As)-9-\{[2,4-difluoro phenyl)methyl]carbamoyl $\}-4$ methyl-6, 8-dioxo-3,4,6,8,12.12 a-hexahydro-2Hpyridol [1',2':4,5] pyrazino [2,1-b] [1,3] oxazin -7-olate (Figure1). It contains a sodium moiety having the formula $-\mathrm{C}_{20} \mathrm{H}_{18} \mathrm{~F}_{2} \mathrm{~N}_{3} \mathrm{NaO}_{5}$ having an exact mass of $441.36 \mathrm{~g} / \mathrm{mol}$. It is light yellow to white powder in looks, being slightly water soluble $[17,18]$.

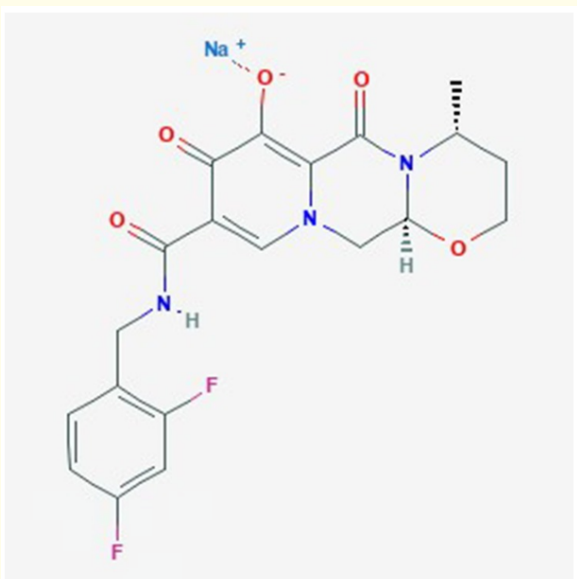

Figure 1: Courtesy ref no-18-DTG molecular structure (adapted from PubChem).

\section{Pharmacodynamics}

DTG represents a strong integrase inhibitor which uses divalent cations $\left(\mathrm{Mg}^{2+}\right)$ for coupling with the enzymatic area of viral integrase. With its structure it can penetrate the active and recently emptied enzymatic pocket where it binds deeper in than previous drugs. This yields a > stable and lasting bond as compared to other precursor integrase inhibitors so that its dissociation constant (mean dissociation constant $2.7 \times 10^{-6} \mathrm{~s}^{-1}$ ) is slower as compared to raltegravir (RAL) or elvitegravir (EVG)-22 and $71 \times 10^{-6} \mathrm{~s}^{-}$ ${ }^{1}$ ),respectively. It also possesses lower half -maximal inhibitory concentration (IC50) for HIV-1 of $1.6 \mathrm{nM}$ compared to 3.3 and $6 \mathrm{nM}$ for RAL as well as EVG respectively [19,20].

\section{Pharmacokinetics}

After ingesting DTG peaks within 2 - 3 hrs. Low, medium and high fat meals enhance DTG's AUC by 33\%, 41\% and 66\% respectively. 
The median time to maximal concentration $\left(\mathrm{T}_{\max }\right)$ is $2.5 \mathrm{hrs}$. It is tightly protein bound (99\%) in plasma and has a medium distribution volume of 17L. Elimination half life is roughly $14 \mathrm{hrs}$ that lets once daily dosing to get used. It gets excreted mainly through faeces (64\%) and urine (31\%). DTG gets metabolized by UGT1A1 (main path) as well as CYP3A (minor path) but does not inhibit UGT1A1 or inhibit or induce any CYP enzymes, hence has a limited drug-drug interaction profile. But it is a substrate of BCRP, P-gp, UGT1A3 as well as UGT1A9 [21].

\section{Lamivudine (3TC)}

Lamivudine is a cytidine analogue having a chemical formula (2R, cis) -4-amino-1-(2 hydroxymethyl-1,3oxathio-lan-5-yl) (1H)-pyrimidin-2-one, formulated as a sulphur salt. Its molecular formula is $\mathrm{C}_{8} \mathrm{H}_{11} \mathrm{~N}_{3} \mathrm{O}_{3} \mathrm{~S}$ having an exact mass of $229.3 \mathrm{~g} / \mathrm{mol}$. It looks like an off white to white crystal which is water soluble having a melting point of $160-162^{\circ} \mathrm{C}[22,23]$ (Figure2).

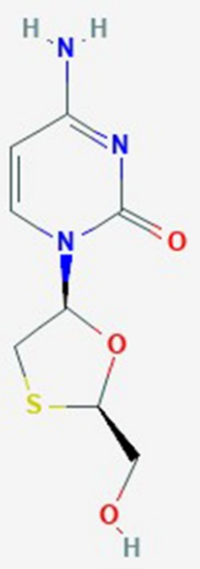

Figure 2: Courtesy ref no-23-3TC molecular structure (adapted from PubChem).

\section{Pharmacodynamics}

3TC competitively inhibits HIV-1 reverse transcriptase by incorporating its active triphosphorylated form in to the viral DNA leading to chain termination. It possesses perfect activity against both HIV-1 as well as HIV-2 virus with an EC50 value of120nm for HIV-1 as well as HIV-2 in cell lines that include PBMC's monocytes. 3TC possess action against hepatitis B virus though monotherapy correlated with resistance formation fast $[24,25]$.

\section{Pharmacokinetics}

After oral intake 3TC gets immediately absorbed with good bioavailability (about 87\%) which is not significantly influenced by meal intake, with a $\mathrm{T}_{\max }$ ) of $1 \mathrm{hr}$.It distributes basically with a peak time of about $3.2 \mathrm{hrs}$ as well as volume of distribution of 96L. Its half-life of 13 - 19 hrs helps in once /day dosage. It does not undergo metabolism in liver and gets excreted mainly via the kidneys, thus dose adjustments are needed in the setting of compromised renal function. It shows linear pharmacokinetics, reaching steady state by day 15 of intake $[25,26]$.

\section{Clinical efficacy}

This DTG/3TC combination was initially analyzed for Efficacy, safety as well as tolerance in the proof of concept PADDLE (Pilot Antiretroviral Design with Dolutegravir 3TCivudinE) study carried out in Argentina. 20 ARV naive subjects having HIV-1RNA < 100,000 copies/mL, CD4 cell count $/ \mathrm{mm}^{3}$, no evident genotypic resistance to Lamivudine as well as with negative hepatitis B surface antigen (HBsAg) at screening got recruited. Median age was 34yrs and 19subjects being male. Primary end point was the percentage of patients having HIV-1RNA $<50$ copies/mL at week 48 . All the recruited subjects finished 48 wks of the study but for 1 patient (who committed suicide) [27].

Evaluation of intention to treat revealed $90 \%$ reached the primary end point by week 48 (per protocol viral suppression rate bei ng 95\%) which included $3 / 4$ subjects who were later observed to have a baseline viral load $>100,000$ copies $/ \mathrm{mL}$. Rapid fall in viral loads was seen in all subjects possessing a $\mathrm{VL}<50$ copies $/ \mathrm{mL}$ by wk 8 . The lone subject who later had viral failure resuppressed without any alteration in therapy off study at wk 48.Median CD cell count change was by 267 cells $/ \mathrm{m}^{3}$ from baseline. Therapy was well tolerated in total and sticking to therapy was $100 \%$ by self report and confirmed by pill counts.

ACTG5353 was a multicenter phase II single arm study of DTG/3TC combination for ARV therapy naive subjects having HIV VL's between 1000 and 500,000 copies/ml with no CD4 count restrictions and no main resistance mutations to NRTIs, NNRTIs or INSTIs. Recruited persons (120) had a median age of 30 yrs were mainly male (87\%) with racial /ethnic composition that had blacks (40\%), Hispanics (28\%) and Asians (2\%). 31\% of subjects had HIV VL's $>100,000$ copies/mL. Median baseline CD4 count was387 cells/ $\mathrm{mm}^{3}$. Primary end point was the percentage of subjects having $\mathrm{VL}<$ 
50 copies/mL at wk 24 utilizing the FDA snapshot algorithm. Secondary outcomes were safety, tolerance evaluation, alteration in CD4 cell count, resistance emerging and examining effectiveness by baseline VL's (< or >100,000copies/ml). DTG values were also estimated for finding the association to study results.

Primary outcomes of the study got achieved in 90\% (108/120) of persons. Results were comparable irrespective of baseline VL's. 5 (4\%) had virologic non-success and 7 (6\%) had no virologic data in the analysis window, virologic failure in 3 subjects took place following early virologic repression and was believed to have been secondary suboptimal sticking to regimen as seen by DTG serum values $(5 \mathrm{ng} / \mathrm{ml}$ at 1 or more time points). The M184V (NRTI resistance) and R263 R/K (INSTI resistance) mutations occurred in 1 subject. In total, the DTG/3TC regimen was well tolerated with no need of discontinuing study because of side effects. Self reported adherence was high with $90 \%$ of subjects documenting adherence religiously [28].

2 Similar multinational, randomized, double blind, non inferiority phase III studies carried out in 192 centres in 21 countries (GEMINI -1 and GEMINI-2) examined DTG/3TC as compared to DTG/tenofovir disoproxil fumarate (TDF)/Emtricitabine (FTC) for therapy of ARV naive subjects. Recruited subjects had to have HIV VL's $<500,000$ copies $/ \mathrm{mL}$, and no major mutations resulting in resistance to NRTIs, NNRTIs or PIs. Those women who had child bearing potential and were on approved contraception modalities were also Recruited. Total of 1441 subjects were randomized 1:1 to either study arm (719 in 2 drug and 722 in 3-drug regimen grps but with 3 and 5 individuals in every group not getting a single dose of drug, respectively). Demographics as well as baseline clinical properties were identical among the groups studied, median age was 33yrs, main subjects were male (85\%) and white $(68 \%)$. Most subjects at baseline had VL's $<100,000$ copies/ml (80\%) and CD 4 count $>200$ cells $/ \mathrm{mm}^{3}$ (92\%). Primary end point was the percentage of subjects having $\mathrm{VL}<50$ copies/mL at wk 48 utilizing the FDA snapshot algorithm with the aim of showing non inferior virologic efficiency with a margin of $-10 \%$. Secondary end point was the percentage of subjects having $\mathrm{VL}<50$ copies/ml at wk 24 , time in wks for reaching $\mathrm{VL}<50$ copies/ml as well as alteration in $\mathrm{CD} 4$ cell count from baseline to wk 48 . Safety end points were incidence as well as how severe side effects and percentage of subjects discontinuing therapy secondary to safety events. Renal as well as bone biomarkers were examined at baseline, wk24 as well as wk48.
In GEMINI -1, the Primary end point in the intention to treat exposed individuals was $90 \%(320 / 356)$ in the 2 drug group and 93\% (332/358) in the 3-drug group. In the same way in GEMINI-2,93\% (335/360) in the 2 drug group and 94\% (337-359) in 3 -drug group managed to achieve the Primary end points. Median time to3TC possesses viral suppression was admirable and seen within 29days. Both studies met the previously set non inferiority criteria for virologic efficiency. On combined evaluation, virologic non response (by definition snapshot analysis as $\geq 50$ copies $/ \mathrm{mL}$ at wk 48) was observed in 3\% (20/716) in the 2 drug group and $2 \%$ $(13 / 717)$ in the 3 -drug group.

10 individuals (1\%) met criteria for virological withdrawl (6 in the 2 drug group and 4 in the 3-drug group). virological withdrawl was met if a $2^{\text {nd }}$ and consecutive VL met any of the reduction in baseline of $<1 \log ^{10}$, unless level was $<200$ by wk 12; VL $>200$ after level was $<200$ copies/mL by wk 12; VL > 200 at or after wk 24; or rebound (VL $>200$ after level was $<200$ copies/ml). Genotypic and phenotypic resistance testing did not show any reverse transcriptase or integrase mutations in these subjects.

In total, the combined results in GEMINI -1 and GEMINI- 2 trials were similar in subjects with greater ( $>100,000$ copies $/ \mathrm{mL}$ ) and lower VL $(\leq 100,000$ copies $/ \mathrm{mL})$. Identical virological response was also observed for subjects with CD4 count $>200$ cells $/ \mathrm{mm}^{3}(93 \%$ in both groups). Low virological response was seen in those having CD4 count $<200$ cells $/ \mathrm{mm}^{3}$ (79\% in 2drug group and $93 \%$ in the 3-drug group group), but was believed to be not related to effectiveness of the treatment regimens as were alterations in health quality of life assessment (EQ-5D5L health utility scales) [29].

Safety profile as well as toxicity Of DTG/3TC in phase III trials

In GEMINI -1 and GEMINI-2 2 trials side effects were revealed in 76\% (543/716) in 2 drug group and 81\% (579/717) in 3-drug group; with comparable safety as well as tolerance in both treatment regimens. Drug-associated side effects were lower in 2 drug group and mainly due to low drug -associated grade1 nausea. Most side effects were not serious and commonly were headache, diarrhea and nasopharyngitis. Side effects affecting discontinuation were identical in the 2 groups. Neuropsychiatric side effects associated with suicidal ideation as well as behavior happened in $2 \%$ in every group. 2 deaths took place 1 in each drug group, but as per the researchers they were not correlated to study drugs.

Citation: Kulvinder Kochar Kaur., et al. "Utilization of Dolutegravir and Lamivudine as Combination as A Single Tablet in Therapy of HIV-1 Infection". Acta Scientific Microbiology 3.3 (2020): 01-08. 
As far as secondary outcomes, alteration in biomarkers associated with kidney and bone safety advocated 2 drug group was more favourable. On the contrary, significantly less enhancement of lipids was seen in the 3-drug group with known effects on kidney, bone and cholesterol. Health related quality of life (QOL) scores was same and high at baseline for both groups and remained high at 48 wks.

Special safety profile as well as toxicity conditions

DTG

Utilization in pregnancy

A National Institute of health that they gave funds for observational study on birth outcomes in pregnant women on cART in Botswana found a probable enhanced chances of neural tube defects on DTG received at time of conception [30]. Since that time an upgradation of therapy guidelines have been made so that risks as well as benefits of therapy using DTG can be shared among women of childbearing potential as well as their providers [31].

Little enhancement of serum creatinine by inhibition of renal transporter OCT2 occurs. The enhancement is especially observed within the $1^{\text {st }} 4$ wks of use but doesn't depict actual reduction in glomelular filtration rate (GFR) or renal function [32].

\section{TC-Resistance barrier as well as mutation}

3TC possesses wide action against both HIV-1 as well as HIV2 virus. On substituting methionine for valine at amino acid position 184 (M184V or M184I) of the reverse transcriptase pol gene of HIV1 resistance forms. The mutation gets easily selected for and appears after many days following exposure to 3TCmonotherapy or suboptimal adhering to regimens having this agent. But the M184I mutation decreases HIV multiplication and total viral fitness; thus good clinical optimal might still be seen in patients with 3TC-resistant strains of HIV1 which continue 3TC therapy in combination with other regimens especially those which include tenofovir to which these strains might have hyper susceptibility $[30,33]$.

\section{Hepatitis B}

3TC possesses anti viral action against Hepatitis B virus (HBV), severe acute exacerbation of Hepatitis might take place in HBV/ HIV coinfected patients, that have utilized 3TC containing regimens and omit it. Hence close monitoring of these subjects is needed '[34].
Drug interactions

DTG

In contrast to other classes of ARV drugs, INSTIs posess a low chance for Drug Interactions. However DTG, has various important Drug Interactions needing understanding. Coadministration of trivalent cations like iron, calcium, aluminium as well as magnesium markedly interfere with DTG absorption. One can reduce these by taking DTG $2 \mathrm{hrs}$ before or $6 \mathrm{hrs}$ following medicines ingestion or substances that have these agents. Drugs inducing CYP3A4 as well as transporters UGT1A1 and P-glycoprotein would decrease DTG amounts. These are rifampin, carbamazepine and EFV hence these shouldn't be given with DTG (daily dosed). When rifampin, carbamazepine coadministration is essential, dosing frequency of DTG needs to be enhanced twice a day for overcoming this.

Conversely Pharmacokinetics of metformin as well as dofelitide get changed when administration is done with DTG and thus ending in enhanced amounts of both drugs. Thus recommendation is to strict to a dose of $1 \mathrm{~g}$ metformin only with simultaneous use. Use of dofelitide with DTG is contraindicated [35].

\section{TC}

Minimal Drug Interactions Are seen with 3TC. Sorbitol possessing products can cause a dose dependent reductions in Cmax and AUC of 3TC on coadministration. Hence these drugs need avoidance in patients receiving 3TC [36].

\section{Regulation}

DOVATO got approved by US FDA in April 2019 for treating HIV1 infection in ARV naive adults with no known viral mutations correlated with resistance to separate components.

On July 3, 2019, EMA gave marketing authority to DOVATO for treating HIV-1 infection in ARV naive adults as well as adolescents $>12 \mathrm{yrs}$ age with weight min of $40 \mathrm{~kg}$ with no known suspected resistance to integrase inhibitor class / 3TC. More applications are worldover pending.

\section{Routes of administration}

DTG/3TC needs to be taken as single tab daily orally with/without food. It is not recommended as a fixed dose tab if creatinine clearance is $<50 \mathrm{ml} / \mathrm{min}$. On administration with rifampin or carbamazepine, extra dose of $50 \mathrm{mg}$ of DTG (as a single agent) needs to be taken $12 \mathrm{hrs}$ from the $1^{\text {st }}$ dose of DTG/3TC [17]. 


\section{Conclusions}

DTG/3TC is a good efficient NRTI sparing combination as the $1^{\text {st }}$ therapy regarding treating HIV infection. Studies that have analyzed DTG/3TC as far as sustenance of virologic suppression for those who have had experience of treatment earlier are continuing, and it is anticipated that they will turn out to have just similar efficiency as observed with DTG/ rilprivine (RPV) which was the $1^{\text {st }}$ approved 2 drug regimen as far as sustainance therapy is concerned.

Both DTG as well as 3TC represent potent antiretroviral agents having good tolerance profiles as well as minimal drug interaction potential. Clinical trials have demonstrated that this regimen has good efficacy in those having great VL's $(100,000$ - 500,000 copies / $\mathrm{mL}$ ) and low CD4 count $\left(<200\right.$ cells $\left./ \mathrm{mm}^{3}\right)$ and the worries about treatment emerging viral resistance for treatment failing are not justified as when resistance to 3TC occurs, resistance to DTG occurring is very rare because of high resistance barrier. Still those who offer therapy, might not want to give DTG/3TC to patients whose chances of adhering to therapy appear poor or suboptrimal.

Some patient categories might not be fully represented or eliminated in the late phase clinical trials of DTG/3TC that includes women, or those who have a very very high VL (> 500,000copies/ $\mathrm{mL}$ ) are essential to understand. These are important to think of using test as well as treat policies, children $<12$ yrs of age /weight $<40 \mathrm{~kg}$, those having coincidental hepatitis B infection, with resistance mutations to NRTI and or INSTI class of AVRs as well as those having renal insufficiency (GFR $<50 \mathrm{~mL} / \mathrm{min}$ ). This influences whether one can generalize the prelicensure studies and emphasize on certain populations where $>$ data is required that points to neural tube defects taking place at low but significantly > rate in pregnant women who had been exposed to DTG compared to other comparator Antiretroviral agents and the rate in the general population, there is continuous worry regarding its use for women of childbearing potentials [31,37]. In the same manner Neuropsychiatric adverse effects taking place at both short term as well as long term complications have been reported with DTG therapy, that includes enhancement of depressive disorders with suicides are to be taken into account by those who prescribe [38]. By eliminating the NRTIs-abacavir (ABC) as well as tenofovir (TDF or TAF), DTG/3TC excludes agents which have been correlated with long term toxicities, that are CVS disease, renal and bone side effects.
But elimination of tenofovir mainly TDF also adds weakness with DTG/3TC combination, in that, that is the regimen acceptable for HIV/HBS coinfected subjects since 3TC is has marked resistance development chances and the good effect of TDF on decreasing lipids, mainly cholesterol gets removed.

Important is INSTI resistance was not checked in GEMINI studies although the product label of DTG/3TC says that it is only indicated in the ones without known INSTI mutations. This is not going to be meaningful clinical practice since prevalence of transmitted (prior to treatment) as INSTI resistance continues to be very $\operatorname{low}(1.2 \%)$ [39].

Availability of generic 3TC helps DTG/3TC to get into the ARV market with a decreased price that is about $26 \%$ lower than that of bictegavir/ TAF /FTC and almost 35\% lower than cost of bictegavir/cobicistat. that was coformulated with TAF/ FTC.THIS might get acceptable effect on use as well as including this drug on therapy formulations [17].

In the coming future, with the expected long acting ARV formulations, to be introduced, the era of the daily dosed STR in the therapy of choice might be seen as the coming of its end. Till it occurs, the approval of an efficacious as well as having good tolerance 2 drug STR for ARV-naive PLWH is a very effective development that has literally changed the scenario and a v welcome combination as a single tablet.

The Swedish Reference Group for Antiviral Therapy (RAV) published recommendations for the treatment of HIV infection most recently in 2017. An expert group under the guidance of RAV here provides updated recommendations. The most important updates in the present guidelines are the following: (a) The risk of HIV transmission through condomless sex from individuals with fully suppressed HIV viral load is effectively zero. (b) Pre-exposure prophylaxis (PrEP) is recommended for groups with a high risk of HIV infection. (c) Since the last update, two new substances have been registered: bictegravir and doravirine. (d) Dual treatment may be an alternative in selected patients, using lamivudine + dolutegravir or lamivudine + boosted darunavir/atazanavir. As with previous publications, recommendations are evidence-graded in accordance with the Oxford Centre for Evidence Based Medicine. This document does not cover treatment of opportunistic infections and tumours [40]. 


\section{Bibliography}

1. Panel on Antiretrovirals Guidelines for Adults and Adolescents. "Guidelines for the use of Antiretroviral agents in HIV-1 -infected Adults and Adolescents". Department of Health and Human Services (2019).

2. World Health Organization. Consolidated Guidelines on the Use of Antiretroviral Drugs for Training and Preventing HIV1 -infection (2016).

3. Saag MS., et al. "Antiretroviral drugs for Treatment and Prevention of HIV-1 -infection in adults :2018 recommendations of the International Antiretroviral Society-USA Panel". Journal of the American Medical Association 32094 (2018): 379-396.

4. European AIDS Clinical Society. European Guidelines for Treatment of HIV-positive Adults in Europe (2017): 2.

5. Min S., et al. "Antiviral activity, safety and Pharmacokinetics/ Pharmacodynamics of dolutegravir as 10days monotherapy in HIV-1 -infected Adults". AIDS 25 (2011): 1737-745.

6. Kozal MJ., et al. "A nucleoside -and ritonavir sparing enzyme containing atanazir plus raltegavir in in Antiretroviral Treatment-naive HIV-1 -infected patients: SPARTAN study results". HIV/AIDS Clinical Trials 13.3 (2012): 119-130.

7. Carr A., et al. "A syndrome of lipoatrophy, lactic acidaemia and liver dysfunction associated with HIV nucleoside analogue therapy: contribution to protease inhibitor -related lipodystrophy syndrome". AIDS 14 (2000): F25-F32.

8. Mallai SA., et al. "Contribution of nucleoside analogue reverse transcriptase inhibitors to subcutaneous fat wasting in patients with HIV infection". AIDS 14 (2000): 1309-1316.

9. Allavena C., et al. "Efficacy and tolerability of a nucleoside reverse transcriptase inhibitor-sparing combination of lopinavir/ ritonavir and efavirenz in HIV-1 infected patients". Journal of Acquired Immune Deficiency Syndromes 39 (2005): 300-306.

10. Libre JM., et al. "Phase III SWORD 1 and 2: switch to DTG+RPV maintains virologic suppression through 48wks [abstract 44LB]". Program and abstracts of the 2017 Conference on Retroviruses and Opportunistic infections (2017).

11. Raffi F., et al. "Once daily dolutegravir versus raltegravir in antiretroviral-naive adults with HIV infection :48wks results from randomised double blind non inferiority SPRING 2 study". Lancet 381 (2013): 735-743.
12. Walmsley SL., et al. "Dolutegravir plus abacavir, Lamivudine for the treatment of HIV 1 infection". The New England Journal of Medicine 369 (2013): 1807-1818.

13. Cahn P., et al. "Dual therapy with lopinavir and ritonavir plus Lamivudine versus triple therapy with lopinavir and ritonavir plus two nucleoside reverse transcriptase inhibitors in Antiretroviral therapy- naive adults with HIV 1 infection (FLAMINGO) :48 wks results from the randomised open-label, noninferiority GARDEL trial". The Lancet Infectious Diseases 14.7 (2014): 572-580.

14. Raffi F., et al. "Dolutegravir efficacy at 48weeks in key subgroups of the treatment--naive HIV-1 -infected individuals in three randomised trials". AIDS 29 (2015): 167-174.

15. Dovato (Dolutegravir/ Lamivudine) [prescribing information]. Research Triangle Park, NC: ViV Healthcare (2019).

16. "Dolutegravir". National center for biotechnology information". Pub chem Compound Database, US. National Library of Medicine (2019).

17. Girrpuard MP., et al. "The cost effectiveness and budget impact of 2 drug Dolutegravir/ Lamivudineregimens for the treatment--naive HIV-1 - infection in the United States". Clinical Infectious Diseases 62 (2016): 784-791.

18. Pubchem. Dolutegravir (2019).

19. Podany AT., et al. "Comparitive clinical Pharmaco-kinetics and Pharmacodynamics of HIV-1 integrase strand transfer inhibitors". Clinical Pharmacokinetics 56.1 (2017): 25-40.

20. Kandel C and Walmsley SL. "DTG-A review of the pharmacology, efficacy and safety in the treatment of HIV". Drug Design, Development and Therapy 9 (2015): 3547-555.

21. Cottrell ML., et al. "Clinical Pharmaco-kinetics, Pharmacodynamics and Drug Interaction profile of the integrase inhibitor Dolutegravir". Clinical Pharmacokinetics 52.11 (2013): 981994.

22. "Lamivudine" National center for biotechnology information. Pub chem Compound Database, US. National Library of Medicine (2019).

23. Pubchem. Lamivudine (2019).

24. Eron JJ., et al. "Treatment with Lamivudine, zidovudine, or both in HIV-1 -positive patients with 200-500 CD4+ cells per cubic millimeter. North American HIV-1working party". The New England Journal of Medicine 333 (1995): 1662-1669. 
25. Moore KHP Pharmacokinetics (PK) of Lamivudine (3TC) phosphorylation on peripheral blood mononuclear cells (PBMC) from HIV-1 - infected patients[abstract]". 12th International Conference on AIDS (1998).

26. Paredes R., et al. "In vivo fitness cost of the M184V mutation in multidrug resistant human immunodeficiency virus type 1 in the absence of Lamivudine". Journal of Virology 83 (2009): 2038-2043.

27. Cahn P., et al. "Dolutegravir/ Lamivudine as initial therapy in HIV-1 - infected ARV naive patients, 48weeksresult of the PADDLE (Pilot Antiretroviral Design with Dolutegravir 3TCivudinE) study". Journal of the International Neuropsychological.

28. Taiwo B., et al. "ACTG5353: A pilot study of Dolutegravir plus Lamivudine for initial Treatment of human immunodeficiency virus type 1 (HIV-1)- infected participants with HIVRNA <500,000copies/ml". Clinical Infectious Diseases 66.11 (2018): 1689-1697.

29. Cahn P., et al. "Dolutegravir plus Lamivudine versus Dolutegravir plus tenofovir disoproxil fumarate and Emtricitabine in t Antiretroviral- naive adults with HIV-1 - infection(GEMINI -1 and GEMINI-2 ):week 48 results from 2 multicenter, randomized ,double blind, non inferiority phase 3 trials". Lancet 393.10167 (2019):143-155.

30. Gao QA., et al. "The same mutation that encodes low-level human immunodeficiency virus type 1 resistance to 2'3'dideoxyi-nosine and 2'3'-dideoxycytidine confers high-level resistance to the (-)enantiomer of the 2'3'-dideoxy- 3'thiacytidine". Antimicrob Agents Chemother 37 (1993): 1390.

31. Panel on Antiretrovirals Guidelines for Adults and Adolescents. "Guidelines for the use of Antiretroviral agents in HIV1 -infected Adults and Adolescents with HIV". Department of Health and Human Services (2019).

32. GutierrezF., et al. "Renal tubular transporter mediated interactions of HIV drugs: implications for patient management". AIDS Reviews 16 (2014): 199-212.

33. Gao WY., et al. "Divergent anti- human immunodeficiency virus activity and anabolic phosphorylation of 2'3'- dideoxy nucleoside analogs in resting and activated human cells". Journal of Biological Chemistry 269 (1994): 12633.

34. Honkoop P "Dolutegravir Acute exacerbation of chronic Hepatitis B virus infection after withdrawl of Lamivudine". Hepatology 32.3 (2000): 635-639.
35. Tivicay (Dolutegravir).

36. Epivir (Lamivudine).

37. Iacobucci G. "HIV: Dolutegravir should be preferred treatment option in all populations, says WHO". British Medical Journal (2019): 14831.

38. CaliholJ., et al. "Dolutegravir and Neuropsychiatric adverse events: a continuing debate". AIDS Reviews 31.14 (2017): 2023-2024.

39. Doyle T., et al. "Integrase inhibitors (INI) genotypic resistance in treatment naive and raltegravir-experienced patiemnts infected with diverse HIV-1 clades". Journal of Antimicrobial Chemotherapy 70 (2015): 3080-3086.

40. Eriksen J., et al. "Antiretroviral Treatment of HIV-1 - infection: Swedish R Aecommendations 2019". Infectious Diseases 11 (2020): 1-35.

\section{Assets from publication with us}

- Prompt Acknowledgement after receiving the article

- Thorough Double blinded peer review

- Rapid Publication

- Issue of Publication Certificate

- High visibility of your Published work

Website: https://www.actascientific.com/

Submit Article: https://www.actascientific.com/submission.php

Email us: editor@actascientific.com

Contact us: +919182824667 